\title{
Assessing the SAfety and FEasibility of Bedside Portable Low-Field Brain Magnetic Resonance Imaging in Patients on ECMO (SAFE-MRI ECMO study) - Study Protocol and First Case Series Experience
}

\author{
Sung-Min Cho ( $\nabla$ csungmi1@jhmi.edu ) \\ Johns Hopkins University School of Medicine \\ Christopher Wilcox \\ Johns Hopkins University School of Medicine \\ Steven Keller \\ Johns Hopkins University School of Medicine \\ Matthew Acton \\ Johns Hopkins University School of Medicine \\ Hannah Rando \\ Johns Hopkins University School of Medicine \\ Eric Etchill \\ Johns Hopkins University School of Medicine \\ Errol L. Bush \\ Johns Hopkins University School of Medicine \\ Haris I. Sair \\ Johns Hopkins University School of Medicine \\ John Pitts \\ Hyperfine \\ Bo Soo Kim \\ Johns Hopkins University School of Medicine \\ Glenn Whitman \\ Johns Hopkins University School of Medicine \\ Kate Giuliano \\ Johns Hopkins University School of Medicine
}

Short Report

Keywords: Portable MRI, ECMO, Safety, Bedside, Neuroimaging, Brain

Posted Date: March 7th, 2022

DOI: https://doi.org/10.21203/rs.3.rs-1409569/v1

License: (1) This work is licensed under a Creative Commons Attribution 4.0 International License. Read Full License 


\section{Abstract \\ BACKGROUND}

To assess the safety and feasibility of imaging of the brain with a point-of-care (POC) magnetic resonance imaging (MRI) system in patients on extracorporeal membrane oxygenation (ECMO). Early detection of acute brain injury (ABI) is critical in improving survival for patients with ECMO support.

\section{METHODS}

Patients from a single tertiary academic ECMO center who underwent head CT (HCT), followed by POC brain MRI exams within 24 hours following HCT while on ECMO. Primary outcomes were safety and feasibility, defined as completion of MRI exam without serious adverse events (SAEs). Secondary outcome was the quality of MR images in assessing ABIs.

\section{RESULTS}

We report 3 consecutive adult patients (median age: 47 years; $67 \%$ male) with veno-arterial $(n=1)$ and veno-venous ECMO ( $n=2)(V A-$ and VV-ECMO) support. All patients were imaged successfully without SAEs. Times to complete POC brain MRI exams were 34,40 , and 43 minutes. Two patients had ECMO suction events, resolved with fluid and repositioning. Two patients were found to have an unsuspected acute stroke, well visualized with MRI.

\section{CONCLUSIONS}

Adult patients with VA- or VV-ECMO support can be safely imaged with low-field POC brain MRI in the intensive care unit, allowing for the assessment of presence and timing of $\mathrm{ABI}$.

\section{Introduction}

Despite rapidly expanding use, ${ }^{1}$ the therapeutic potential of ECMO is limited by significant morbidity such as acute brain injury (ABI), a devastating complication. ${ }^{2,3}$ Protocolized neuromonitoring improves the detection of $\mathrm{ABI}$ and may limit its sequelae with timely management. ${ }^{4}$ However, early diagnosis of $\mathrm{ABI}$ is difficult due to an inability to safely transport patients and lack of available transport personnel. Even when possible, HCT (currently the only imaging modality used), has limited sensitivity for detecting ischemic $A B I$. The gold standard for diagnosing $A B I$, conventional systems rely on high-strength magnetic fields (1.5-3T) incompatible with extracorporeal life support circuits.

Recent advances in low-field, portable MRI technology enable acquisition of clinically meaningful imaging in the presence of ferromagnetic materials. ${ }^{5}$ Low-field MRI (0.064T) produces less than 5 Gauss beyond the 5 foot safety zone of the magnet's center. This is a marked reduction in the magnetic footprint compared with conventional MRI, making it feasible for ICU use without adverse events. ${ }^{5}$ Phantom and animal studies demonstrate the safety and compatibility of portable MRI without deleterious magnetic force or heating of the ECMO circuit or its components. ${ }^{6}$

The current study seeks (1) to evaluate the safety and feasibility of low-field point-of-care (POC) MRI to obtain brain imaging in ECMO patients and (2) to use those MRI images to assess for the presence of $A B I$. We hypothesized low-field MRI can produce high quality neuroimages superior to HCT to enable bedside detection of $\mathrm{ABI}$.

\section{Methods}

The Johns Hopkins Medicine Institutional Review Board approved this study (IRB00285716). Consent was obtained from a legally authorized representative as enrolled patients were unable to provide consent.

\section{Study Design/Population}

This study presents the first 3 patients of an ongoing prospective observational study of POC MRI in adults with VA- or VV-ECMO. Exclusion criteria included weight over $200 \mathrm{~kg}$, active pregnancy, and contraindications to 1.5T MRI other than ECMO such as incompatible implants.

\section{Study Procedure}

HCT exams were routinely performed on these patients as part of a standardized neuromonitoring protocol ${ }^{4}$, with the addition of POC MRI within 24 hours utilizing a $64 \mathrm{mT}$ Swoop ${ }^{\circledR}$ MR imaging system (Hyperfine, Inc., Guilford, CT). The MR system was positioned into the patient's room with all ICU equipment outside the magnet's $5 \mathrm{G}$ safety line (Supplemental Fig. 1). Once the patient's bed was aligned with the MRI head coil, 4 trained individuals, including a perfusionist, respiratory therapist, intensivist, and nurse slid the patient into position using a lift-and-slide maneuver. Pads placed around the patient's head prevented any motion. Vital signs were monitored continuously during the exam. Resultant images were read by a neuroradiologist (H.I.S.) who was blinded in clinical information. 
Medical equipment was positioned appropriately to allow the portable MRI to be situated at the head of the bed. The patient was maintained as flat as possible while positioning, at the direction of the team leader. A research coordinator and intensivist physician monitored vital signs, ECMO flow, and positioning of the cannula and the endotracheal tube.

Serious Adverse Events (SAEs): The following changes were considered SAEs: i) change in mean arterial pressure (MAP) of $\pm 20 \%$; ii) decrease in ECMO flow rate of $10 \%$; or iii) decrease in oxygen level $\left(\mathrm{SpO}_{2}\right)$ of $10 \%$ from baseline.

\section{Outcomes}

Primary outcomes were safety and feasibility, defined as completion of the POC MRI exam without SAEs. The secondary outcome was the quality of MR images compared to HCT images, determined by a board-certified neuroradiologist (H.I.S.).

\section{Results}

\section{Patient Characteristics}

We report the first 3 consecutive patients (median age: 47 years; $67 \%$ male) who had both HCT and POC MRI exams performed during ECMO support. Two patients were supported with VV-ECMO due to COVID-19 acute respiratory syndrome (ARDS), and one was supported with VA-ECMO due to cardiogenic shock from massive pulmonary embolism (Table 1).

Table 1

Patient characteristics and adverse events during portable brain MRI scan

\begin{tabular}{|c|c|c|c|c|c|c|c|c|c|c|c|}
\hline & $\begin{array}{l}\text { Age } \\
\text { (yrs) }\end{array}$ & Sex & BMI & $\begin{array}{l}\text { ECMO } \\
\text { indication }\end{array}$ & $\begin{array}{l}\text { Cannulation } \\
\text { strategy }\end{array}$ & $\begin{array}{l}\text { Neurologic } \\
\text { symptoms }\end{array}$ & $\begin{array}{l}\text { MRI } \\
\text { time }\end{array}$ & HCT finding & $\begin{array}{l}\text { MRI } \\
\text { finding }\end{array}$ & $\begin{array}{l}\mathrm{ABI} \\
\text { management }\end{array}$ & $\begin{array}{l}\text { Adverse } \\
\text { Events* }\end{array}$ \\
\hline 1 & 47 & Male & 30.5 & $\begin{array}{l}\text { COVID-19 } \\
\text { ARDS }\end{array}$ & $\begin{array}{l}\text { Fem-IJ (V- } \\
\text { V) }\end{array}$ & $\begin{array}{l}\text { Coma } \\
\text { under } \\
\text { sedation }\end{array}$ & $\begin{array}{l}40 \\
\text { minutes }\end{array}$ & $\begin{array}{l}\text { Acute left } \\
\text { occipital } \\
\text { ischemic } \\
\text { stroke with } \\
\text { hemorrhagic } \\
\text { conversion }\end{array}$ & $\begin{array}{l}\text { Acute } \\
\text { left } \\
\text { occipital } \\
\text { ischemic } \\
\text { stroke }\end{array}$ & $\begin{array}{l}\text { Anticoagulation } \\
\text { held and } \\
\text { restarted with } \\
\text { serial imaging } \\
\text { studies }\end{array}$ & $\begin{array}{l}\text { One self- } \\
\text { limited } \\
\text { ECMO } \\
\text { suction } \\
\text { event }\end{array}$ \\
\hline 2 & 45 & Male & 32.5 & $\begin{array}{l}\text { COVID-19 } \\
\text { ARDS }\end{array}$ & $\begin{array}{l}\text { Fem-IJ (V- } \\
\text { V) }\end{array}$ & $\begin{array}{l}\text { Coma } \\
\text { under } \\
\text { sedation }\end{array}$ & $\begin{array}{l}43 \\
\text { minutes }\end{array}$ & $\begin{array}{l}\text { No acute } \\
\text { findings }\end{array}$ & None & Not applicable & None \\
\hline 3 & 55 & Female & 27.1 & $\begin{array}{l}\text { Cardiogenic } \\
\text { Shock with } \\
\text { PE }\end{array}$ & $\begin{array}{l}\text { Fem-Fem } \\
(\mathrm{V}-\mathrm{A})\end{array}$ & $\begin{array}{l}\text { Coma } \\
\text { under } \\
\text { sedation }\end{array}$ & $\begin{array}{l}34 \\
\text { minutes }\end{array}$ & $\begin{array}{l}\text { No acute } \\
\text { findings }\end{array}$ & $\begin{array}{l}\text { Acute } \\
\text { right } \\
\text { basal } \\
\text { ganglia } \\
\text { ischemic } \\
\text { infarct }\end{array}$ & $\begin{array}{l}\text { Anticoagulation } \\
\text { held and } \\
\text { restarted with } \\
\text { serial imaging } \\
\text { studies }\end{array}$ & $\begin{array}{l}\text { Frequent } \\
\text { ECMO } \\
\text { suction } \\
\text { events, } \\
\text { received } \\
\text { intravenous } \\
\text { fluids; } \\
\text { Improved } \\
\text { with } \\
\text { repositioning } \\
\text { patient* }\end{array}$ \\
\hline
\end{tabular}

\section{Safety and Feasibility}

All patients were imaged successfully, without significant changes in MAP, ECMO flow, and $\mathrm{SpO}_{2}$ (SAEs). MRI exams were successfully completed in all three patients in 34, 40 and 43 minutes. Two patients had ECMO cannula suction events, which resolved with fluid bolus and repositioning (Table 1). Clinical Presentation and Imaging Findings

Patient \#1: A 47-year-old male without significant medical history, cannulated with VV-ECMO for refractory COVID-19 ARDS. Patient had a routine HCT at day 15 of ECMO showing an acute ischemic stroke in the left occipital lobe with small hemorrhagic conversion. Ischemic stroke was confirmed with portable MRI, however, it was more difficult to diagnose small hemorrhagic conversion with MRI vs. HCT (Fig. 1).

Patient \#2: A 45-year-old male without a significant medical history, cannulated with VV-ECMO for severe COVID-19 ARDS. Routine HCT at day 12 was conducted as our standard neuromonitoring protocol, followed by a POC MRI, neither of which showed any acute intracranial abnormalities.

Patient \#3: A 55-year-old female with a recent pulmonary right upper lobectomy for primary lung adenocarcinoma with massive pulmonary embolism postoperatively, who required VA-ECMO for right heart failure. Although HCT was normal at day 4, the subsequent POC MRI within 24 hours showed an acute right basal ganglia ischemic stroke.

\section{Quality of Images}


MR images were able to detect discernible pathologies with good quality. Two patients had acute strokes (\#1, \#3), which were well visualized by POC $\mathrm{MRI}$, one of which was not seen on HCT (patient \#3).

\section{Discussion}

This study evaluated the feasibility and safety of portable POC brain MRI in patients with ECMO support, which represents a "first-in-human" investigation of this technology in the ECMO setting. POC MRI was shown to be safe and feasible with all exams completed in under 43 minutes without SAEs. In ECMO patients where MRI at 1.5T is contraindicated and transportation for HCT can be dangerous and require significant human resources, POC MRI allows early ABI detection, which is critical in improving ECMO survival. ${ }^{4}$ As demonstrated, POC MRI detected new ischemic strokes, one of which was not visualized with HCT, suggesting POC MRI may be a good bedside diagnostic tool for cerebral ischemia. As demonstrated in patient \#1, the detection of hemorrhagic conversion in ischemic stroke needs further experience and validation, especially, when hemorrhages are small.

This early experience provides insight into how to further improve care of ECMO patients by optimizing duration of the MRI exam time and patient positioning. In this study, four MR sequences were obtained in a relatively long scan time: T1-weighted, T2-weighted, fluid-attenuated inversion recovery, and diffusion-weighted imaging with an automatically calculated apparent diffusion coefficient map. Eliminating one or two of the sequences that are mirrored by others could decrease duration of the MRI exam, such as the T1. Patient body habitus, as seen in our patients, made positioning in the scanner challenging. As observed, 2 patients had temporary ECMO suction events that appeared to be the result of insufficient support of the thoracolumbar spine leading to flexion at the groin. Repositioning the body as flat as possible, thereby preventing flexion of the spine between the bed and the scanning platform resolved the suction events, making it likely that the femoral drainage cannula position was compromised.

Overall, our study demonstrates that POC MRI in the ECMO patient is feasible and may offer logistic as well as diagnostic advantages over HCT, particularly given that early detection and timely intervention of $\mathrm{ABI}$ is of paramount importance in improving outcomes in the care of ECMO patients. Accessible POC MRI has the potential to markedly improve our ability to diagnose subclinical ABI and to immediately alter clinical management with the goal of mitigating injury.

\section{Conclusions}

Adult patients with VA- and VV-ECMO support can be safely imaged with a low-field POC brain MR at the bedside in the ICU setting, allowing for the assessment and treatment of $A B I$.

\section{Declarations}

\section{Author's contributions}

S.M.C. and G.W. designed the study. S.M.C., G.W., B.S.K., C.W., S.K., M.A., and H.R. conducted the experiments, and performed data extraction. S.M.C prepared the first draft. H.I.S. reviewed the MR images and H.I.S., C.W. and J.P. prepared figures. C.W. the table. All authors read, revised, and approved the final manuscript.

\section{Source of Funding}

This study was funded by Hyperfine, Inc.

S.M.C. is funded by NHLBI 1K23HL157610.

S.P.K. is funded by NHLBI 5K08HL14332.

\section{Availability of Data}

Authors agree to make the data available.

\section{Ethical Approval and Consent to Participate}

The Johns Hopkins Medicine Institutional Review Board approved this study (IRB00285716). Consent was obtained from a legally authorized representative.

\section{Consent for publication}

N/A.

\section{Conflicts of Interest/Disclosures}

All other authors declare that the research was conducted in the absence of any commercial or financial relationships that could be construed as a potential conflict of interest. 


\section{References}

1. McCarthy FH, McDermott KM, Kini V, et al. Trends in U.S. Extracorporeal Membrane Oxygenation Use and Outcomes: 2002-2012. Semin Thorac Cardiovasc Surg. 2015;27(2):81-8. doi:10.1053/j.semtcvs.2015.07.005

2. Cho SM, Canner J, Caturegli G, et al. Risk Factors of Ischemic and Hemorrhagic Strokes During Venovenous Extracorporeal Membrane Oxygenation: Analysis of Data From the Extracorporeal Life Support Organization Registry. Crit Care Med. Nov 2020;doi:10.1097/CCM.0000000000004707

3. Cho SM, Canner J, Chiarini G, et al. Modifiable Risk Factors and Mortality From Ischemic and Hemorrhagic Strokes in Patients Receiving Venoarterial Extracorporeal Membrane Oxygenation: Results From the Extracorporeal Life Support Organization Registry. Crit Care Med. Oct 2020;48(10):e897-e905. doi:10.1097/CCM.0000000000004498

4. Ong CS, Etchill E, Dong J, et al. Neuromonitoring detects brain injury in patients receiving extracorporeal membrane oxygenation support. J Thorac Cardiovasc Surg. Oct 30 2021;doi:10.1016/j.jtcvs.2021.09.063

5. Sheth KN, Mazurek MH, Yuen MM, et al. Assessment of Brain Injury Using Portable, Low-Field Magnetic Resonance Imaging at the Bedside of Critically III Patients. JAMA Neurol. Sep 2020;doi:10.1001/jamaneurol.2020.3263

6. Etchill E, By S, Giuliano K, et al. SAFETY AND FEASIBILITY OF PORTABLE MRI WITH ECMO (SAFE-ECMO STUDY). presented at: Society of Critical Care Medicine; 2022;

\section{Figures}

\section{Figure 1}

Point-of-care brain MRI images and corresponding CT images.

Images $A, F, K, P$ are apparent diffusion coefficient MRI, images $B, G, L, Q$ are diffusion weighted imaging $M R I$, images $C, H$, M, R are fluid-attenuated inversion recovery $\mathrm{MRI}$, and images $\mathrm{E}, \mathrm{J}, \mathrm{O}, \mathrm{T}$ are CT scans. Patient 1 (Images A-J) demonstrated acute occipital stroke with hemorrhagic conversion as well as two acute frontal lobe strokes denoted by red arrows. Patient 2 (K-O) had normal MRI and CT scan. Patient 3 (P-T) had an acute right basal ganglia stroke (red arrow) confirmed on other MRI sequences and not identified on CT.

\section{Supplementary Files}

This is a list of supplementary files associated with this preprint. Click to download.

- SupplementalMaterial.docx 